\title{
Resonance states of open quantum dots
}

\author{
Yongjiang Wang \\ Department of Physics, University of Toronto, Toronto, Canada \\ Ningiia Zhu \\ Centre for the Physics of Materials, Department of Physics, McGill University, Montréal, Québec, H3A 2T8 Canada \\ Jian Wang \\ Department of Physics, The University of Hong Kong, Pokfulam Road, Hong Kong \\ Hong Guo \\ Centre for the Physics of Materials, Department of Physics, McGill University, Montréal, Québec, H3A 2T8 Canada
}

(Received 1 May 1995; revised manuscript received 13 July 1995)

\begin{abstract}
We have computed the spectra of resonance states for several open quantum dot systems. These states are identified using the electron dwell time. The statistics of the spectra are exactly the same as that of the corresponding closed system, even when the level widths are comparable with the average spacing. In particular, for a regular structure, e.g., an open rectangular quantum dot, the resonance state level spacing satisfies the Poisson distribution. For an irregular structure, e.g., an open Sinai billiard, we found that the spacings satisfy the GOE or GUE statistics depending on whether an external magnetic field is applied. Thus in this regime of ballistic transport, the statistics of resonance transmission contains characteristics of the corresponding intrinsic quantum level distribution. [S0163-1829(96)04124-0]
\end{abstract}

\section{INTRODUCTION}

Electrons traversing through ultrasmall semiconductor microstructures give rise to a fascinating regime of quantum transport. ${ }^{1}$ When the system size is reduced to near or below the mean free path of charge carriers, a variety of phenomena associated with quantum interference can be easily observed. These include the study of the universal conductance fluctuations in the mesoscopic regime, ${ }^{2}$ the Aharonov-Bohm effects, ${ }^{3}$ the quenching of the quantum Hall effects, ${ }^{4}$ and the junction resonances. ${ }^{5}$ Recently, in an interesting experiment, Marcus et $a l^{6}$ measured the conductance of a twodimensional stadium-shaped quantum dot, connected to the outside by two point contacts. Large aperiodic conductance fluctuations were observed. It is well known that the motion of a classical particle is chaotic when it bounces off the walls inside a closed stadium-shaped box. ${ }^{7}$ Thus by measuring conductance of an irregular quantum dot, one has the possibility of investigating quantum chaotic scattering. From a theoretical point of view, the semiconductor structures thus provide a testing ground for theories and ideas in the intriguing field of quantum chaos. ${ }^{7,8}$

Although there is no rigorous and unique definition of quantum chaos, it generally refers to quantum systems whose classical analog is chaotic, such as a stadium-shaped quantum dot, ${ }^{9}$ or quantum systems whose eigenvalue spectrum satisfies Dyson ensembles, ${ }^{10}$ such as the Anderson model. For closed systems, quantum chaos is studied by solving the one-particle Schrödinger equation, and characterizing the statistics of the energy levels. ${ }^{10}$ For open systems one is dealing with a scattering problem of charge carriers by some peculiar boundary, and an important characteristic is the observed system dependent conductance fluctuations. ${ }^{1,12}$ So far quantum chaotic scattering has been studied from several directions such as the investigation of statistical properties of the scattering matrix,${ }^{11,12}$ the study of the distribution function of the values of transmission coefficients, ${ }^{13}$ and the statistics of level widths in quantum dots. ${ }^{14}$

In this paper we present a direct calculation of the statistical properties of quantum scattering states or the resonance states which are responsible for the junction resonances often observed in multiprobe quantum-dot-based systems. ${ }^{5}$ In particular we will study the manifestation of quantum level spacing statistics. There are several motivations of this work. First, while resonance states play an important role in resonant transmission in quantum devices, direct calculations of these states for two-dimensional structures have been quite limited due to technical difficulties, ${ }^{15}$ and the statistics of these states have not been directly computed. Second, a major part of our understanding of "quantum chaos" is based on the eigenenergy level statistics of closed systems. Thus we believe it is desirable to directly relate the statistics to the observed conductance fluctuations of the open system. When a billiard structure becomes open by connecting it to external leads, the eigenenergy levels become resonance state levels. The resonant transmission often observed experimentally can be viewed as mediated by these states. Obviously the statistics of these state levels is directly related to the correlation of the conductance fluctuation. ${ }^{16}$ While the resonance state levels are shifted in energy from those of the corresponding bound states ${ }^{17}$ of the closed system, recently we have shown that the one-to-one correspondence between the resonance and the bound states holds at low energies. ${ }^{18}$ However, at higher energies, or in situations where the width of the resonance state levels is comparable to the average level spacing, such one-to-one correspondence is less clear. Thus an inter- 
esting question arises: does the level spacing of resonance states satisfy the same statistics as those of the corresponding bound states? While we expect, especially in the case of weak coupling between the leads and the quantum dot, ${ }^{12}$ that the resonance states satisfy the same statistics, it is nevertheless extremely difficult to directly compute enough levels for open quantum dot systems for a meaningful statistical analysis; and it is not at all clear for the strong coupling case since there the level smearing is quite large.

Our direct numerical calculation shows (see below) that the resonance state spectra for several open quantum dot systems satisfy exactly the same statistics as that of the corresponding closed systems, even when the level widths are comparable with the average spacing. In particular, for a regular structure, e.g., an open rectangular quantum dot, the resonance state level spacing satisfies the Poisson distribution. For an irregular structure, e.g., an open Sinai billiard, we found that the spacings satisfy the GOE or GUE statistics depending on whether an external magnetic field is applied. It turned out that the energy levels of the resonance states are very close to those energies where transmission maxima or minima occur. Thus in this regime of ballistic transport, one can achieve the understanding that the statistics of resonance transmission contains the characteristics of the corresponding intrinsic quantum level distribution.

The paper is organized in the following way. In the next section the method and results are presented, and a short summary is in the last section.

\section{METHOD AND RESULTS}

We study transmission of electrons through two kinds of quantum dot structures. Each dot is connected by two probes where electrons come from one probe, scatter inside the dot, and transmit to the other probe or reflect back. We investigated both a strong and weak coupling situation between the probes and the dots. In the weak coupling case tunneling barriers are added at the connections between the probes and the dots, while no tunnel barriers are present in the strong coupling case. The first structure is a rectangle quantum dot which is "regular" in the sense that the motion of a classical particle inside the dot is not chaotic. The second structure is a Sinai billiard which is "irregular" since the motion of classical particles inside is chaotic. For the irregular structure we also study the consequence of an external magnetic field. The structures are shown in the insets of Figs. 3 and 4.

Typically in the study of quantum level statistics, one needs to compute thousands of levels in order to have good statistical averages. However, for the scattering problem considered here, this is practically impossible to do. We also note that experimentally ballistic transport in semiconductor nanostructures involves relatively low energy. Thus we shall focus on the statistical properties of the resonance states at low energies. For the Sinai billiard, we have put three hard disks inside the quantum dot. By randomly changing the positions of these disks we were able to generate an ensemble with uncorrelated resonance patterns. This allowed us to determine the resonance states (see below) at relatively low energy and ensemble average to obtain reasonable statistics. ${ }^{8}$ For the regular structure we simply varied the width of the quantum wires connecting the dot to generate an ensemble for averaging.
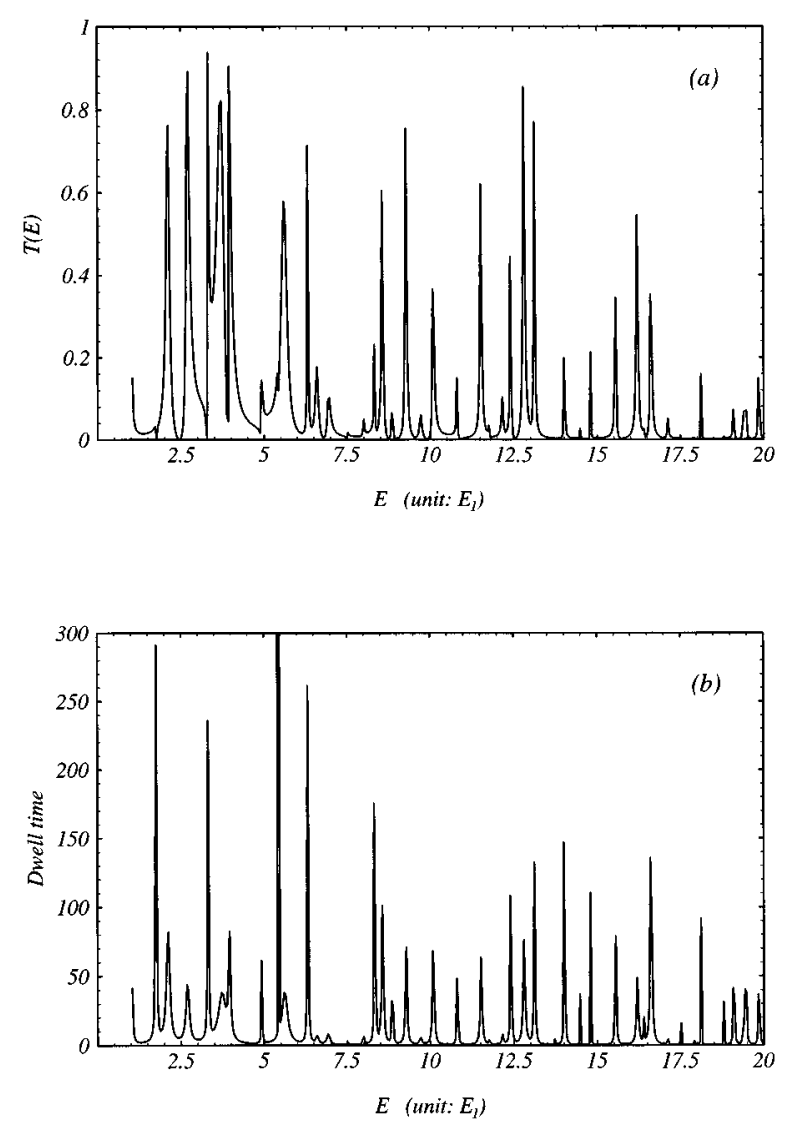

FIG. 1. Typical transmission coefficient and dwell time of a Sinai billiard quantum dot versus incident energy without magnetic field, in the tunneling regime where the leads couple weakly with the quantum dot. (a) The transmission coefficient $T(E)$; (b) the dwell time $\tau_{d}$. In this case the peaks in $\tau_{d}$ coincide with the peaks of $T(E)$ very well.

The resonance states can be identified by calculating the dwell time of the incident electron. ${ }^{18}$ Dwell time ${ }^{19}$ measures the duration an electron spends in the scattering region of a structure. Thus if transport is mediated by resonances, we expect much longer dwell times when the incident electron is at the energy of a resonance state than the times for other incident energies. At junction resonances where $\tau_{d}$ is peaked, the transmission coefficients take extremal values due to resonance transmission (see below). As we have shown in a recent paper, ${ }^{18}$ this "operational" method is able to unambiguously identify resonance states regardless of whether the quantum dot is strongly or weakly coupled to the quantum wires.

The dwell time $\tau_{d}$ is defined ${ }^{19}$ as the ratio of the number of particles within the region of interest $(\Omega)$ to the incident flux $J$,

$$
\tau_{d}=\int_{\Omega}|\Psi(\vec{r})|^{2} d \vec{r} / J
$$

In our case, $\Omega$ is the quantum dot region. The quantum scattering problem is solved using a finite-element numerical scheme ${ }^{20,21}$ for the case of Sinai billiards, and using a modematching method ${ }^{22}$ for the regular dot. With the solution of 

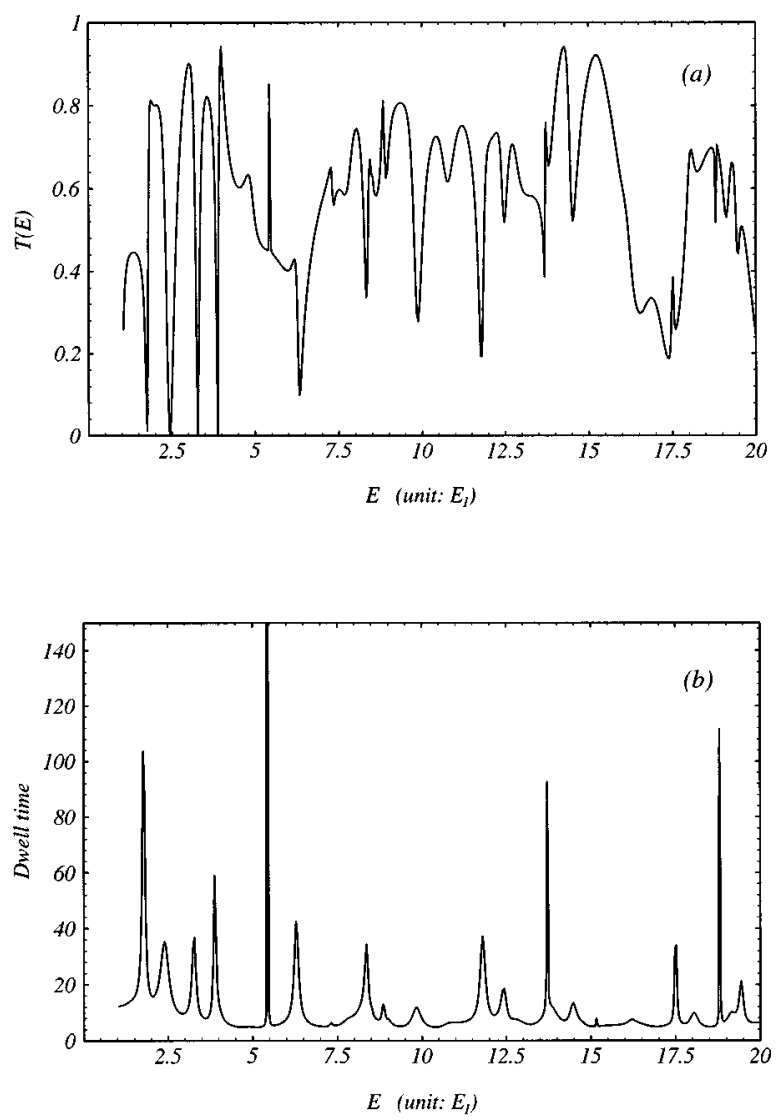

FIG. 2. Typical transmission coefficient and dwell time of a Sinai billiard quantum dot versus incident energy without magnetic field, in the transmissive regime without tunneling barriers where the leads couple strongly with the quantum dot. (a) The transmission coefficient $T(E)$; (b) the dwell time $\tau_{d}$. Notice that in this case a peak in $\tau_{d}$ may coincide with either a peak or a valley in $T(E)$.

the wave functions, we can compute the dwell time $\tau_{d}$. The solution also gives the transmission coefficients.

For the Sinai billiard, we have fixed the quantum dot dimension to be $3000 \AA \times 3790 \AA$ (see insert of Fig. 3). The width of the probes is $1500 \AA$ with the two probes at right angle. The radius of the disks is fixed at $375 \AA, 405 \AA$, and $500 \AA$ with their centers randomly chosen but without the disks overlapping each other. We have used about 3300 grid points to discretize the quantum dot region for the solution of the scattering problem. ${ }^{21}$ For the tunneling case, the heights of the barriers are kept three times higher than the incident electron energy. We have varied the incident electron energy in steps of $0.01 E_{1}$ to $0.04 E_{1}$ where $E_{1}$ is the first subband energy of the quantum wires (the probes). The resonance state levels are computed up to $26 E_{1}$ in the tunneling case, and up to $41 E_{1}$ in the transmissive case.

We first present results for the case of zero magnetic field. Figures 1(a) and 2(a) show typical transmission coefficients $T(E)$ as a function of the incident electron energy for a Sinai billiard with and without the tunneling barriers. In both cases the resonant pattern is clearly seen. We have found that in the tunneling regime the maxima of the dwell time coincide quite well with the transmission peaks. However, in the transmissive case the maxima of the dwell time may coincide with the minima of the transmission coefficient, as seen
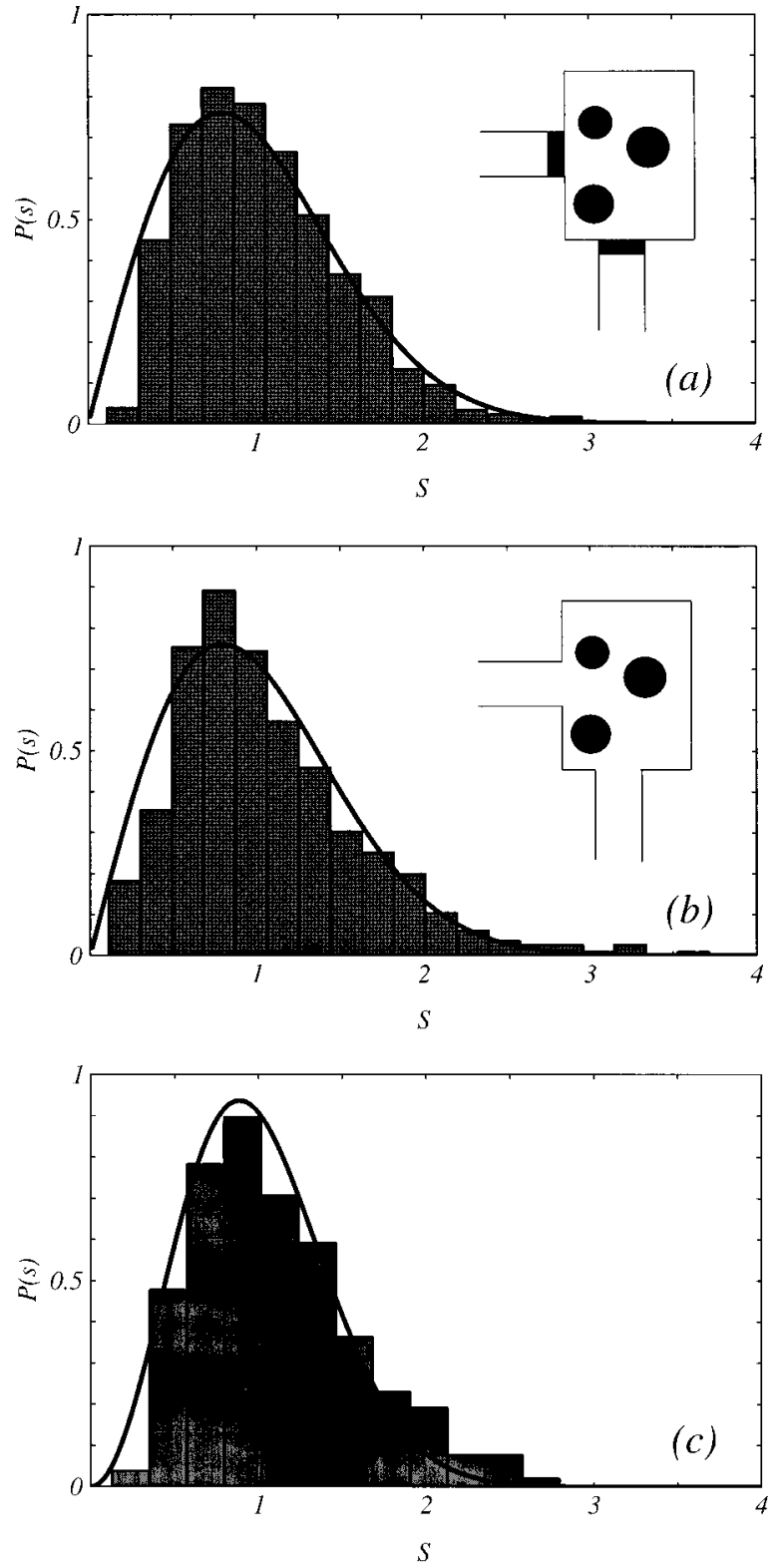

FIG. 3. Distribution function of the resonance state level spacings for the ensemble of Sinai billiard quantum dots. (a) for the tunneling case without magnetic field where a total of 902 resonance levels were collected from 16 dots below $26 E_{1}$. (b) for the transmissive case without magnetic field where a total of 579 resonance levels were found from 15 dots below $41 E_{1}$. Solid lines are the Wigner distribution function, Eq. (1). Insets: plot of the open Sinai billiard quantum dot with hardwall potential boundaries. (c) for the transmissive case with a uniform magnetic field of $830 \mathrm{G}$. 235 resonance levels were found from eight dots. The solid curve is from the distribution Eq. (2).

previously. ${ }^{18}$ The corresponding curves of $\tau_{d}(E)$ are shown in Figs. 1(b) and 2(b) for the tunneling and transmissive cases, and in the latter case the leads strongly couple with the scattering region. It is clear that the sharpness of $\tau_{d}$ makes the "operational" definition of the resonance states reasonable. ${ }^{18}$ For the transmissive case the peak values of $\tau_{d}$ are smaller than those of the tunneling case, an indication of larger level widths. For a detailed discussion of the nature and origin of the resonance states in open quantum dot sys- 

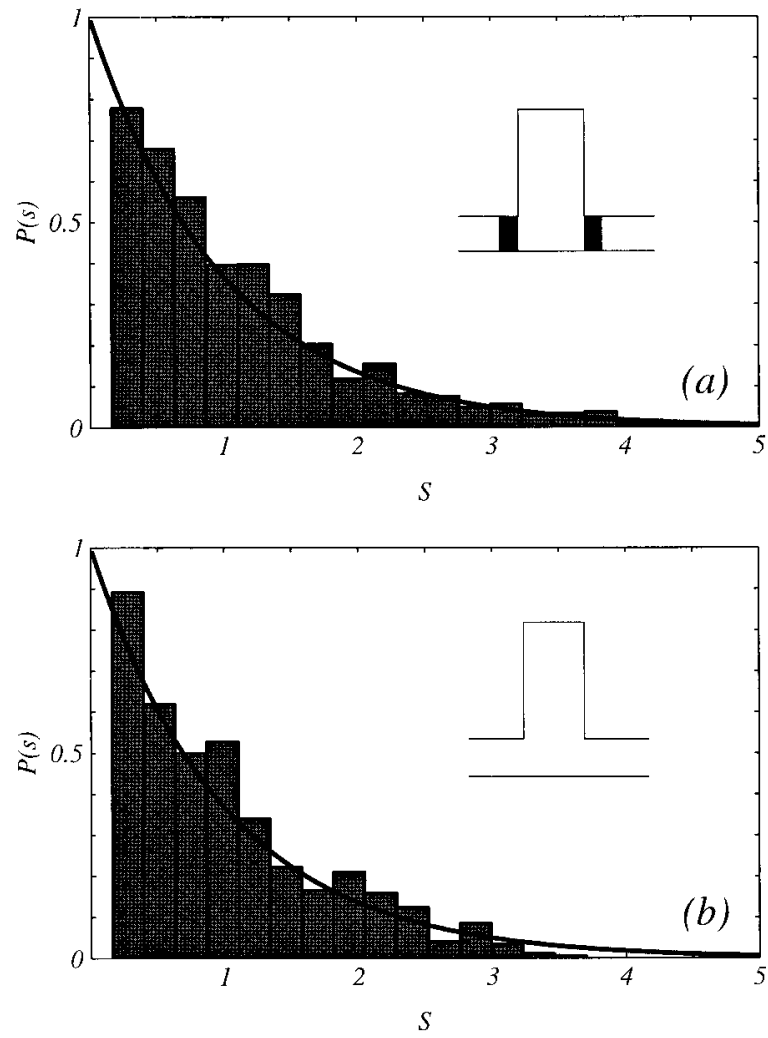

FIG. 4. Distribution function of the resonance state level spacings for the ensemble of regular quantum dots. (a) for the tunneling case where 2562 resonance levels were collected from 42 systems. (b) for the transmissive case where 2535 levels were used. Solid lines are the Poisson distribution function. Insets: plot of the open regular quantum dot with hardwall potential boundaries.

tems we refer interested readers to Ref. 18. In what follows the resonance state levels, $\left\{\epsilon_{i}\right\}$, are taken as the peak positions of the dwell time. ${ }^{18}$

With the energy steps mentioned above we typically found around 50 to 60 resonance state levels with energies less than $26 E_{1}$ in the tunneling case, while only about 35 levels in the transmissive case below $41 E_{1}$. In either case the number of levels is less than an estimate using the Weyl formula for the corresponding closed system. ${ }^{7}$ This indicates that due to the finite level width, especially in the transmissive case, many levels overlap and merge, leading to fewer resonance states in the open structure than the corresponding closed structure. For the tunneling case, we have studied an ensemble of 16 Sinai billiards where a total of 902 resonance states were identified. For the transmissive case, we were able to collect 579 resonance states using 15 billiards. Standard statistical analyses ${ }^{25}$ on these resonance state levels were then performed. ${ }^{10,24,9}$

An interesting quantity is the distribution function of the level spacings. For each member of the billiard ensemble, we first computed the average level spacing $\bar{s}$. The level spacings were then measured in units of $\bar{s}: s_{i} \equiv\left(\epsilon_{i+1}-\epsilon_{i}\right) / \bar{s}$. This was done for each member of the billiard ensemble and all the spacings were then sorted into a list to compute the distribution function. It is well known that for a closed Sinai billiard in zero magnetic field, the statistics of the bound state level spacings belong to the Gaussian orthogonal ensemble (GOE), which is taken as the signal of quantum chaos: ${ }^{7}$

$$
P(s)=\frac{\pi}{2} \operatorname{sexp}\left(\frac{\pi}{4} s^{2}\right) .
$$

Figures 3(a) and 3(b) shows the distribution function for the resonance state levels together with the Wigner distribution (1). In both tunneling and transmissive cases, the overall agreement is very good. We note that due to the finite resolution of the energy scan in our calculation, some resonance states with energies extremely close to others may have been missed for the tunneling case. This leads to the shortage of states in the lowest bin in Fig. 3(a). For the transmissive case, on the other hand, levels extremely close to each other do not occur due to the much larger level widths of each level. In this case our finite resolution in the energy scan does not miss states, hence the agreement with Wigner distribution is better at the lowest energy bin. We thus conclude that the ensemble of open Sinai billiards has resonance states satisfying the same statistics as those of the closed system, whether or not in the tunneling regime. The important message is that although the resonance state levels are shifted in energy from those of the corresponding bound states, and the finite widths of the resonance state levels lead to the merger and overlap of many states, the universal behavior of the level spacing statistics does not change. Hence the fluctuations of the transmission coefficient and conductance [e.g., Figs. 1(a) and 2(a)] in this kind of irregular quantum dot will carry the characteristics of the GOE statistics.

Since in a transport measurement one often uses magnetic field as a control parameter, it is of interest to investigate the resonance states spectra when $B$ is present. When an external uniform magnetic field $B$ is applied, the universality of the level statistics will change. For a closed system the random matrix theory predicts the following distribution when spinorbit scattering is neglected (Gaussian unitary ensemble or GUE),

$$
P_{2}(s)=\frac{32}{\pi^{2}} s^{2} \exp \left(-\frac{4}{\pi} s^{2}\right) .
$$

For the same ensemble of open Sinai billiards as discussed above (no tunneling barriers), we have computed a collection of resonance states for a field strength $B=830 \mathrm{G}$, which corresponds to less than two flux quanta in the scattering region. Similar to the previous situation, due to strong coupling to the leads there are about 30 resonance states identified below energy $41 E_{1}$ where $E_{1}$ is the first subband energy without magnetic field. In Fig. 3(c) the distribution of the resonance state levels is plotted and compared with Eq. (2) above, where a total of 235 levels from eight billiards were included in the analysis. Although the sample is quite small, the agreement with (2) is still reasonable. ${ }^{23}$

To compare with the Sinai billiards, we have computed resonance states of an ensemble of regular quantum dots, as shown in the inset of Fig. 4 and described above. The corresponding bound state levels of the closed dots satisfy a Poisson distribution, as shown by Berry and Tabor: ${ }^{26}$

$$
P(s)=e^{-s} .
$$




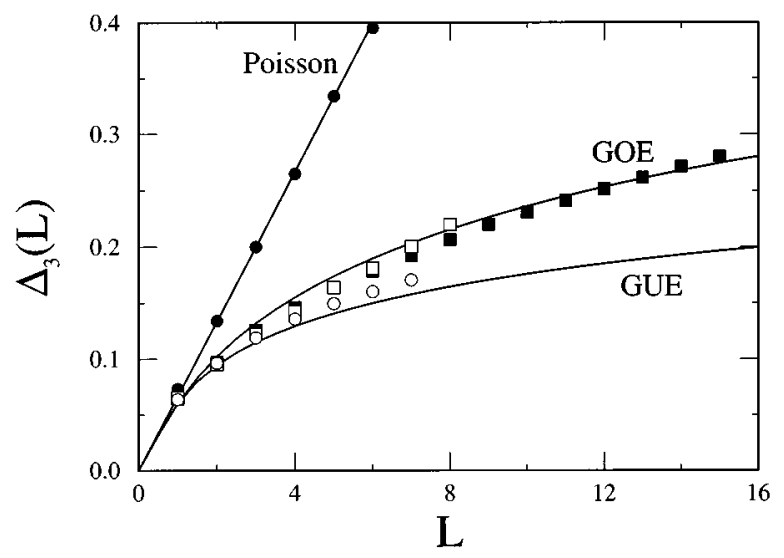

FIG. 5. The spectral rigidity $\Delta_{3}(L)$ as a function of the number of levels $L$. Solid square: from resonance states of the open Sinai billiards in the tunneling regime $(B=0)$. Open square: from the Sinai billiard data in the transmissive case $(B=0)$. Open circle: from the Sinai billiard data with $B=830 \mathrm{G}$. Solid circle: from resonance states of the open regular dots. The solid lines are an analytical formula for different ensembles, from Ref. 28.

For the tunneling case we have collected a total of 2562 resonance state levels from an ensemble of 42 regular systems, and for the transmissive case 2535 levels were collected using 39 structures. Statistical analysis similar to above was carried out. Figure 4 shows the distribution of the resonance state level spacings. The agreement with the Poisson distribution is quite reasonable for both the tunneling and the transmissive cases. Once again the opening of the quantum dot does not change the universal statistics of level spacings.

Another often used measure in studying level statistics is the spectral rigidity $\Delta_{3}$, defined as ${ }^{27,10}$ the mean square deviation of the best local fit straight line to the staircase cumulative spectral density over a normalized energy scale. This quantity measures the long-range rigidity of the level spectrum and is expected to be less sensitive to the possibility of missing a few levels which are very close to other levels due to our finite resolution of the energy scan. Specifically, for a given number $L$ of resonance state levels we computed an average of $\Delta_{3}(L)$ following Ref. 28 and the data are compared with the analytical formula in Ref. 29 from the random matrix theory. The results are shown in Fig. 5 , where the solid lines are from analytical formula. ${ }^{29} \mathrm{In}-$ deed, data of the resonance state levels for Sinai billiards without magnetic field (solid and open squares) agree rea- sonably well with the GOE result, and is far from both the GUE line and the Poisson line. With a finite magnetic field, our numerical data deviates from the GOE line toward the GUE line, as clearly shown in Fig. 5 (open circles). Unfortunately the numerical calculation with a finite $B$ was exceedingly extensive, which prevented us from obtaining enough levels and longer spectra that would improve the statistical fluctuations of $\Delta_{3}$. This is quite similar to the problems encountered in analyzing the bound state spectrum. However, the trend of the data shown indicates the transition from GOE to GUE when a finite magnetic field is present. Finally, we have checked that data for the regular structure, i.e., the rectangle quantum dots (solid circles), agree with the Poisson statistics, ${ }^{30}$ as shown in Fig. 5. These results are completely consistent with the behavior of spacing distributions discussed above.

\section{SUMMARY}

In summary, we have demonstrated, by direct numerical computation, that the spectra of resonance states as determined by the electron dwell time in quantum dots have the same universal statistical properties as those of the corresponding bound states when the quantum dots are closed. In particular, we obtained Wigner distribution of the resonance state spacings for systems with classical chaotic dynamics, and Poisson distribution for classically regular structures. It is rather interesting that even in the very transmissive case, where the resonance states have large level widths, the statistics does not change. We thus conclude that in this regime of the quantum transport, the correlations of the fluctuation in transmission coefficient and conductance will indeed reflect the different level statistics for regular or irregular structures. Theoretically this already has been seen. ${ }^{16}$ In typical experimental situations on submicrometer structures the single particle level spacing is around $0.05 \mathrm{meV}$, thus it can be measured if the temperature is kept less than $500 \mathrm{mK}$. Since experimentally both the tunneling and transmissive cases can be achieved, a measurement on resonant transmission should be able to directly test the resonance state level statistics studied here.

\section{ACKNOWLEDGMENTS}

We thank Zhida Yan and Professor R. Harris for many useful discussions. This work was supported by the Natural Sciences and Engineering Research Council of Canada and le Fonds pour la Formation de Chercheurs et l'Aide à la Recherche de la Province du Québec.
${ }^{1}$ C.W.J. Beenakker and H. van Houten, Solid State Phys. 44, 1 (1991).

${ }^{2}$ C.P. Umbach, S. Washburn, R.B. Laibowitz, and R.A. Webb, Phys. Rev. B 30, 4048 (1984).

${ }^{3}$ See, for example, articles in Physics and Technology of Submicron structures, edited by H. Heinrich, G. Bauer, and F. Kuchar (Springer-Verlag, New York, 1988).

${ }^{4}$ M.L. Roukes, A. Scherer, S.J. Allen, H.G. Craighead, R.M. Ru- then, E.D. Beebe, and J.P. Harbison, Phys. Rev. Lett. 59, 3011 (1987).

${ }^{5}$ M. Büttiker, Phys. Rev. B 38, 12724 (1988); F.M. Peeters, Superlatt. Microstruct. 6, 217 (1989); G. Kirczenow, Phys. Rev. Lett. 62, 1920 (1989); D.G. Ravenhall, H.W. Wyld, and R.L. Schult, ibid. 62, 1780 (1989); Y.J. Wang, J. Wang and H. Guo, Phys. Rev. B 47, 4348 (1993).

${ }^{6}$ C. M. Marcus, A. J. Rimberg, R. M. Westervelt, P. F. Hopkins, 
and A. C. Gossard, Phys. Rev. Lett. 69, 506 (1992).

${ }^{7}$ M. Gutzwiller, Chaos in Classical and Quantum Mechanics (Springer Verlag, New York, 1991).

${ }^{8}$ B.D. Simons, A. Szafer, and B.L. Altshuler, Pis'ma Zh. Éksp. Teor. Fiz. 57, 268 (1993) [JETP Lett. 57, 276 (1993)]; A. Szafer and B.L. Altshuler, Phys. Rev. Lett. 70, 587 (1993); B. D. Simons and B. L. Altshuler, ibid. 70, 4063 (1993).

${ }^{9}$ O. Bohigas, M.J. Giannoni, and C. Schmidt, Phys. Rev. Lett. 52, 1 (1984); T.H. Seligman and J.J.M. Vrbaarschot, in Quantum Chaos and Statistical Nuclear Physics, edited by T.H. Seligman and H. Nishioka, Lecture Notes in Physics Vol. 263 (SpringerVerlag, Berlin, 1986), p. 131.

${ }^{10}$ F.J. Dyson, J. Math. Phys. 3, 140 (1962); 3, 157 (1962); 3, 166 (1962); F.J. Dyson and M.L. Mehta, ibid. 4, 701 (1963); M.L. Mehta and F.J. Dyson, ibid. 4, 713 (1963).

${ }^{11}$ R. Blümel and U. Smilansky, Phys. Rev. Lett. 60, 477 (1988); U. Smilansky, in Chaos and Quantum Physics, edited by M.J. Giannoni, A. Voros, and J. Zinn-Justin (Elsevier, Amsterdam, 1992).

${ }^{12}$ S. Iida, H.A. Weidenmüller, and J.A. Zuk, Ann. Phys. 200, 219 (1990); M.R. Zirnbauer, Nucl. Phys. A560, 95 (1993).

${ }^{13}$ V.N. Prigodin, K.B. Efetov, and S. Iida, Phys. Rev. Lett. 71, 1230 (1993); H.U. Baranger and P.A. Mello (unpublished); R.A. Jalabert, J.-L. Pichard, and C.W.J. Beenakker, Europhys. Lett. 27, 255 (1994).

${ }^{14}$ R.A. Jalabert, A.D. Stone, and Y. Alhassid, Phys. Rev. Lett. 68, 3468 (1992); E.R. Mucciolo, V.N. Prigodin, and B.L. Altshuler, Phys. Rev. B 51, 1714 (1995).

${ }^{15}$ F.M. Peeters, in Science and Engineering of One- and ZeroDimensional Semiconductors, edited by S.P. Beaumont and C.M. Sotomajor Tores (Plenum Press, New York, 1990); M.L. Glasser, W. Jaskólski, F. Garcí a-Moliner, and V.R. Velasco, Phys. Rev. B 42, 7630 (1990).
${ }^{16}$ Yongjiang Wang, J. Wang, H. Guo, and C. Roland, J. Phys. Condens. Matter 6, L143 (1994).

${ }^{17} \mathrm{We}$ assume the capacitance of the quantum dot to be large, thus neglecting the Coulomb blockade effects, i.e., the electrons are assumed to be not interacting.

${ }^{18}$ J. Wang, Yongjiang Wang, and H. Guo, Appl. Phys. Lett. 65, 1793 (1994).

${ }^{19}$ M. Büttiker, Phys. Rev. B 27, 6178 (1983).

${ }^{20}$ C.S. Lent, J. Appl. Phys. 67, 6353 (1990).

${ }^{21}$ Yongjiang Wang, Jian Wang, and Hong Guo, Phys. Rev. B 49, 1928 (1994).

${ }^{22}$ R.L. Schult, D.G. Ravenhall, and H.W. Wyld, Phys. Rev. B 39, 5476 (1989).

${ }^{23}$ It was extremely CPU intensive to compute enough resonance states. For the results presented here, several months of computation were needed on two SGI Power series workstations.

${ }^{24}$ A. Pandey, Ann. Phys. 119, 170 (1979).

${ }^{25}$ The resonance states with their energies defined by the peak positions of $\tau_{d}$ cover a subclass of all the levels of the system. For instance, the true bound states of the system which lie below the transmission threshold of the quantum wire are not included.

${ }^{26}$ M.V. Berry and M. Tabor, Proc. R. Soc. (London) Ser. A 356, 375 (1977).

${ }^{27}$ M.V. Berry, Proc. R. Soc. London Ser. A 400, 229 (1985).

${ }^{28}$ G. Casati, B.V. Chirikov, and I. Guarneri, Phys. Rev. Lett. 54, 1350 (1985).

${ }^{29}$ A. Pandey, in Quantum Chaos and Statistical Nuclear Physics, edited by T.H. Seligman and H. Nishioka, Lecture Notes in Physics Vol. 263 (Springer-Verlag, Berlin, 1986), p. 98.

${ }^{30}$ Although the data of the regular structure agree very well with the Poisson line in this range of $L$, at larger $L$ 's a deviation to the Poisson line is seen in exactly the same fashion as discussed in Ref. 28, which is due to the finite length of the energy spectrum. 